\title{
Room-temperature single charge sensitivity in carbon nanotube field-effect transistors
}

\section{Citation}

Peng, H. B., M. E. Hughes, and J. A. Golovchenko. 2006. "Room-Temperature Single Charge Sensitivity in Carbon Nanotube Field-Effect Transistors." Applied Physics Letters 89 (24) (December 11): 243502. doi:10.1063/1.2399942. http://dx.doi.org/10.1063/1.2399942.

\section{Published Version}

doi:10.1063/1.2399942

\section{Permanent link}

http://nrs.harvard.edu/urn-3:HUL.InstRepos:34334610

\section{Terms of Use}

This article was downloaded from Harvard University's DASH repository, and is made available under the terms and conditions applicable to Other Posted Material, as set forth at http:// nrs.harvard.edu/urn-3:HUL.InstRepos:dash.current.terms-of-use\#LAA

\section{Share Your Story}

The Harvard community has made this article openly available.

Please share how this access benefits you. Submit a story.

\section{Accessibility}




\title{
Room-temperature single charge sensitivity in carbon nanotube field-effect transistors
}

\author{
H. B. Peng \\ Department of Physics, Harvard University, Cambridge, Massachusetts 02138 \\ M. E. Hughes \\ Division of Engineering and Applied Sciences, Harvard University, Cambridge, Massachusetts 02138 \\ J. A. Golovchenko ${ }^{a}$ \\ Department of Physics, Harvard University, Cambridge, Massachusetts and Division of Engineering \\ and Applied Sciences, Harvard University, Cambridge, Massachusetts 02138
}

(Received 10 August 2006; accepted 23 October 2006; published online 11 December 2006)

\begin{abstract}
Electrical current fluctuation studies are reported for coaxial $p$-type and $n$-type single-wall carbon nanotube field-effect transistors (FETs). Abrupt discrete switching of the source-drain current is observed at room temperature. The authors attribute these random telegraph signals to charge fluctuating electron traps near the FET conduction channels. Evolution of the current-switching behavior associated with the occupancy of individual electron traps is demonstrated and analyzed statistically. The result strongly indicates room temperature single charge sensitivity in carbon nanotube FETs, which may offer potential applications for single molecule sensors based on suitably prepared FET devices. (C) 2006 American Institute of Physics. [DOI: 10.1063/1.2399942]
\end{abstract}

Carbon nanotubes are potential candidates for nanoelectronic applications such as field-effect transistors and sensors. ${ }^{1-5}$ The inherent noise of such devices is important yet few studies have addressed noise issues, ${ }^{6-9}$ and these have mainly focused on nanotubes in an "open-to-air" nonpassivated configuration. Here we report on electrical current fluctuations in passivated coaxial $p$ - and $n$-type single-wall carbon nanotube (SWCNT) field-effect transistors (FETs). Room temperature source-drain current switching between quantized levels is observed in these devices, indicating single charge sensitivity of SWCNT FETs. Our results suggest that suitably designed devices may serve as effective single charged biomolecule detectors at room temperature.

SWCNT FETs were fabricated according to a previously described method. ${ }^{10-13}$ Briefly, suspended SWCNTs bridging metal electrodes $(\mathrm{Pt} / \mathrm{Cr})$ were grown across gaps etched through freestanding silicon nitride membranes by chemical vapor deposition, and gate dielectrics and gate electrodes were patterned in a coaxial configuration. ${ }^{11,12}$ p-type FETs were fabricated by coating SWCNTs with a silicon nitride gate dielectric using low-pressure chemical vapor deposition (LPCVD) followed by patterning a metal gate electrode $(\mathrm{Au} / \mathrm{Cr})$ on top. Figure 1(a) shows a schematic of a $p$-type FET structure. For $n$-type FETs, ${ }^{12,13}$ the SWCNTs are coated with a thermally evaporated layer of $\mathrm{Cr}$ (a few nanometer thick), followed by an atomic-layer deposition coating of $\sim 10 \mathrm{~nm}$ of $\mathrm{Al}_{2} \mathrm{O}_{3}$ and a LPCVD coating of silicon nitride $(\sim 100 \mathrm{~nm})$.

Figure 1(b) shows the room temperature source-drain current $I_{D S}$ as a function of gate voltage $V_{g}$ under a sourcedrain bias $V_{D S}=0.2 \mathrm{~V}$ for a $p$-type SWCNT FET. $I_{D S}$ values were also recorded as a function of time at fixed gate voltages and source-drain bias voltages. Discrete switching of $I_{D S}$ is clearly observed [Fig. 1(c)]. We attribute these random-telegraph signals to charge fluctuating electron traps

a)Electronic mail: golovchenko@physics.harvard.edu near the nanotube channel, as has been observed for narrowchannel complementary metal-oxide semiconductor FETs at low temperatures. ${ }^{14}$ Quantized transitions presumably occur when an electron hops from the conducting channel to nearby traps and vice versa, causing the current to switch
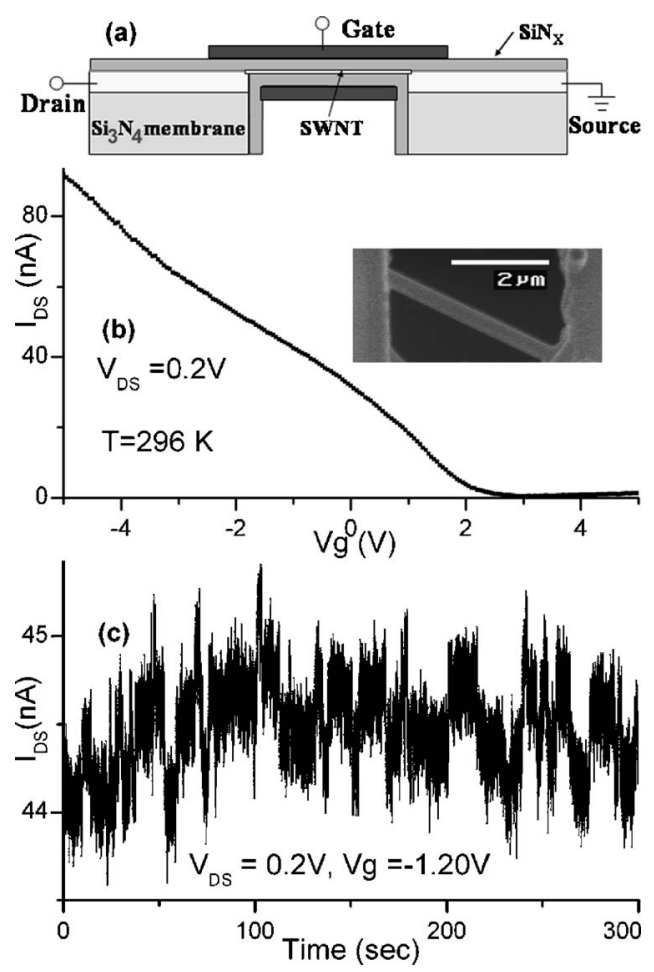

FIG. 1. (a) Schematic of the $p$-type SWCNT FETs. (b) Source-drain current $I_{D S}$ as a function of gate voltage $V_{g}$ at room temperature for a $p$-type FET made by coating a nanotube with $\sim 150 \mathrm{~nm}$ LPCVD silicon nitride in radius as the gate dielectric. Inset: SEM image of the device after the LPCVD coating but before patterning the gate electrode. (c) $I_{D S}$ as a function of time under a source-drain bias $V_{D S}=0.2 \mathrm{~V}$ and gate voltage $V_{g}=-1.2 \mathrm{~V}$ at room temperature using a sampling rate of $300 \mathrm{~Hz}$ and a low-pass filter with a rise-time constant of $3 \mathrm{~ms}$. 

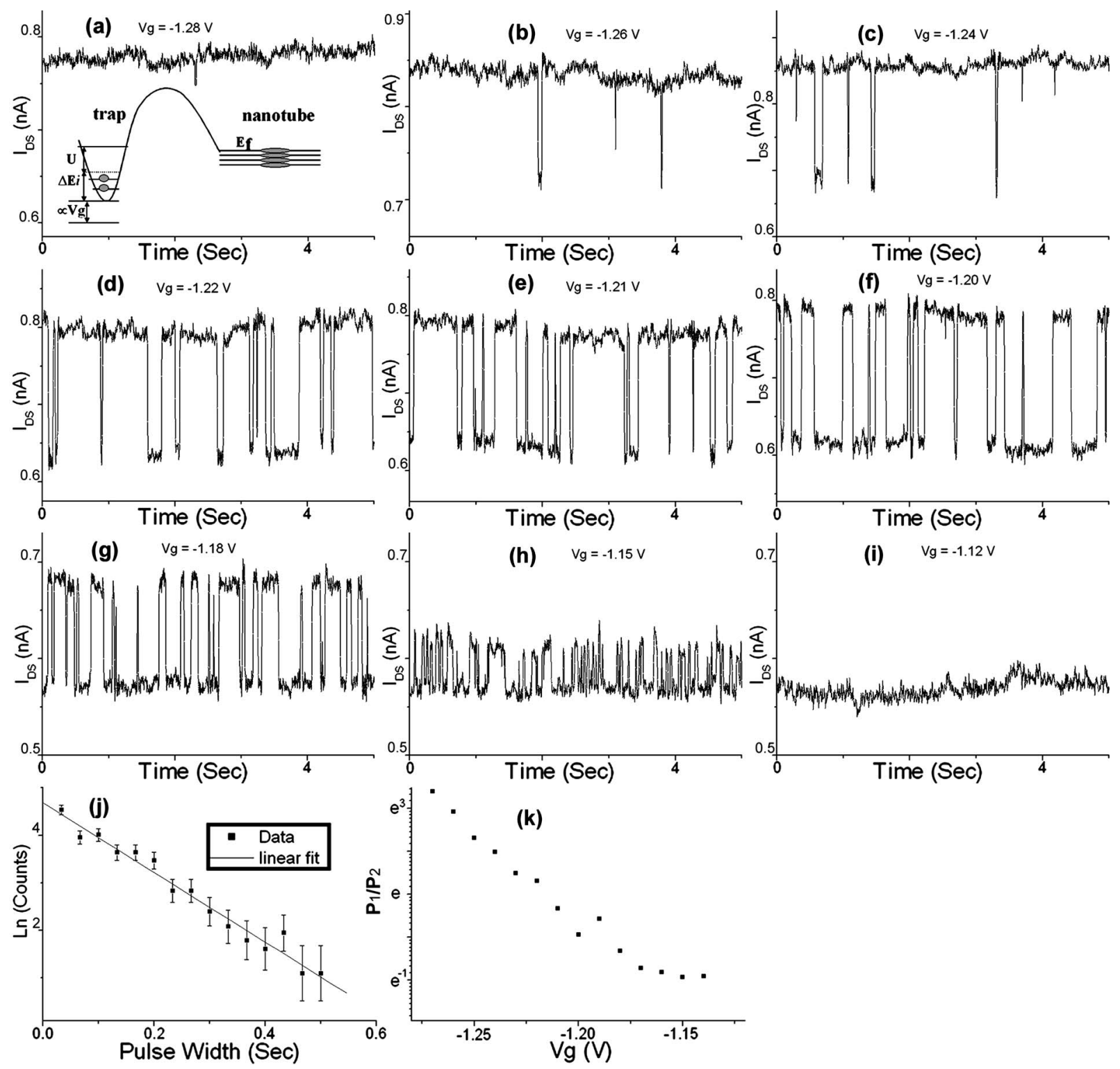

FIG. 2. [(a)-(i)] Source-drain current $I_{D S}$ as a function of time under fixed source-drain bias $V_{D S}=0.2 \mathrm{~V}$ with different gate voltages at a temperature $T$ $=7 \mathrm{~K}$ for the sample shown in Fig. 1. (A notch filter of 60 and $120 \mathrm{~Hz}$ was utilized.) Inset of (a): Energy diagram of electron tunneling between the nanotube and a nearby electron trap. The energy of the trap state consists of the electrostatic potential induced by $V_{g}$, the intrinsic energy spacing $\Delta E_{i}$, and the Coulomb charging energy $U$. (j) Statistical distribution of the pulse width of the upper $I_{D S}$ level at $V_{g}=-1.20 \mathrm{~V}$. (k) Logarithmic-scale plot of the probability ratio between the upper and the lower level $\left(P_{1} / P_{2}\right)$ as a function of gate voltage. Data processing in $(\mathrm{j})$ and $(\mathrm{k})$ is based on $100 \mathrm{~s}$ recordings of the source-drain current.

between discrete levels. The SWCNT FET contains very few electrons in the conducting channel, so it is not surprising that one can observe switching even at room temperature.

At lower temperatures, it is possible to more clearly resolve and study the two level switching (Fig. 2). At a temperature of $T=7 \mathrm{~K}$, as $V_{g}$ sweeps from -1.28 to $-1.12 \mathrm{~V}$, the fluctuating current changes from being dominated by an upper level to a state that spends equal times in an upper and a lower level on average, and finally to a state dominated by the lower level [Figs. 2(a)-2(i)]. These observations are explained by a gate-voltage dependent energy shift of an electron trap near the nanotube. As shown in the inset of Fig. 2(a), the energy difference between the trap and the Fermi level of the SWCNT is varied by the change of $V_{g}$, modulating the occupancy of the electron trap. An electron hops from the nanotube Fermi level to the trap state as the gate-voltage sweeps towards the positive direction, decreasing the number of effective current-carrying electrons and leading to a lower Downloaded 30 May 2007 to 128.103.60.225. Redistribution subject source-drain current. (For an ideal quantum ballistic onedimensional conductor, each current-carrying electron mode gives a conductance of $e^{2} / h \sim 1 / 25.8 \mathrm{k} \Omega$. Hence, it is reasonable that current switching due to the trapping of one electron can be observed. In addition, the Coulomb potential of a charge changing trap may also influence the transmittance of conducting modes in a semiconducting SWCNT.)

Statistically, at a fixed gate voltage, if we assume a constant tunneling rate $\lambda$ from the conducting channel to the trap, the probability for $I_{D S}$ to switch from the upper to the lower level within times $T$ and $T+\Delta T$ (small $\Delta T$ ) should be $P=\lambda \Delta T e^{-\lambda T}$. For a small $\Delta T$, ln P should be linearly dependent on $T$ with slope $-\lambda$. Figure 2(j) depicts the distribution of the pulse widths (of the upper level) at $V_{g}=-1.20 \mathrm{~V}$ based on a $100 \mathrm{~s}$ record of $I_{D S}$. For data analysis, we use $\Delta T$ $=1 / 30 \mathrm{~s}$. The ordinate represents the logarithmic plot of the number of pulses with a width between $t$ and $t+\Delta T$, where $t=m \Delta T$ is the abscissa (with $m$ being an integer). Linear to AIP license or copyright, see http://apl.aip.org/apl/copyright.jsp 

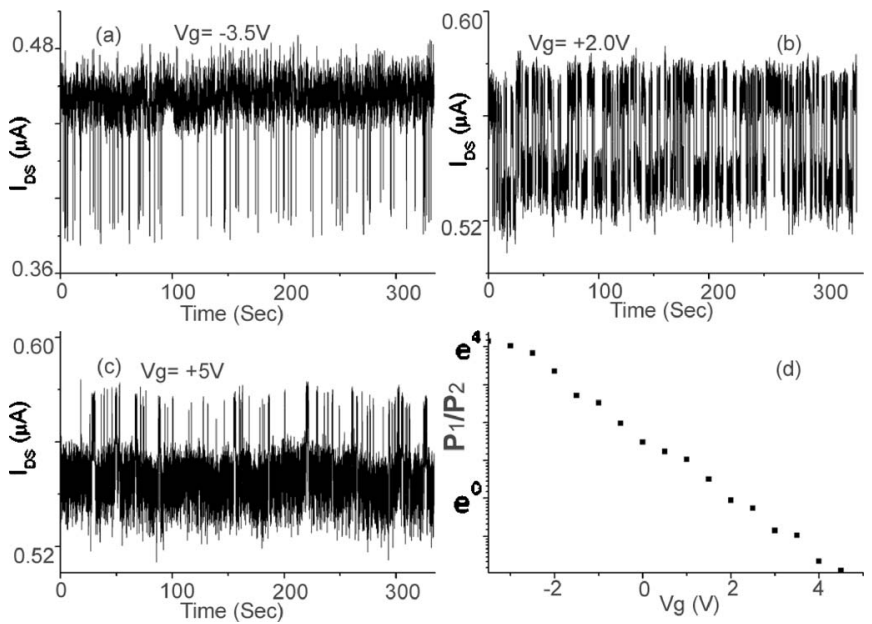

FIG. 3. [(a)-(c)] Source-drain current $I_{D S}$ at room temperature as a function of time under fixed source-drain bias $V_{D S}=-1 \mathrm{~V}$ with different gate voltages for a $n$-type FET made by coating suspended nanotubes with $\sim 1.4 \mathrm{~nm} \mathrm{Cr}$, and then a combination of $20 \mathrm{~nm} \mathrm{Al} \mathrm{O}_{3}$ (by atomic-layer deposition) and $100 \mathrm{~nm}$ silicon nitride (by LPCVD) in radius as the gate dielectrics. The bottom level in (c) shows fluctuations which may be due to the contribution of another two-state switching on a smaller energy scale. (d) Logarithmicscale plot of the probability ratio between the upper and the lower level $\left(P_{1} / P_{2}\right)$ as a function of gate voltage under $V_{D S}=-1 \mathrm{~V}$ for the $n$-type FET.

fitting gives $\lambda_{1}=7.31 / \mathrm{s}$. Since the ratio between the overall duration at the upper level and that at the lower level is near equal at this gate voltage, we expect equivalent tunneling rates: either the electron hops from the SWCNT to the trap, or from the trap to the SWCNT. A similar analysis for the lower level gives nearly identical results as that for the upper level, as expected.

The gate-voltage dependence of the ratio of occupation probabilities for the two levels is obtained by adopting the simple model illustrated in the inset of Fig. 2(a). According to equilibrium statistical mechanics, this probability ratio is given by

$$
P_{1} / P_{2}=\left(g_{1} / g_{2}\right) e^{-\beta\left(E_{f}-E_{t}\right)}=\left(g_{1} / g_{2}\right) e^{-\beta \Delta E},
$$

where $g_{1}$ is the degeneracy of the Fermi level, $g_{2}$ is the degeneracy of the trap state, $E_{f}$ is the Fermi energy, and $E_{t}$ is the trap state energy. ( $E_{t}$ includes contributions of the electrostatic potential induced by $V_{g}$, the intrinsic trap energy, and the Coulomb charging energy.)

Assuming a linear dependence of $\Delta E$ on $V_{g}$, i.e., $\Delta E$ $=c e\left(V_{g}-V_{0}\right)$, where $c$ and $V_{0}$ are constants, $\ln \left(P_{1} / P_{2}\right)$ should be linearly dependent on $V_{g}$. Figure 2(k) shows a plot of $\ln \left(P_{1} / P_{2}\right)$ as a function of $V_{g}$ where the ratio of probabilities is taken as the ratio of times spent at the different current levels. A linear dependence holds in most of the transition region of $V_{g}$, while increasing contributions from more than one trap can account for the deviation from a linear behavior for $V_{g}>-1.15 \mathrm{~V}$.

We also observe current-switching behavior in $n$-type SWCNT FETs. An evolution of the occupancy of a singleelectron trap associated with gate-voltage sweeping is resolved even at room temperature in a $n$-type coaxial FET (Fig. 3). The discrete random telegraph signals approach $\sim 20 \%$ in relative amplitude at room temperature. The switching strongly suggests single-electron sensitivity at room temperature. Figure 3(d) shows the probability ratio of the two levels as a function $V_{g}$, clearly indicating a depen- dence as predicted by Eq. (1). Linear fitting gives a slope $k$ $=-\beta e c-=-0.791 / \mathrm{V}$, so our constant $c=0.021$. Using a model of a coaxial cylinder with the gate electrode as the outer equipotential surface and the Cr-coated SWCNT as the inner surface and assuming the SWCNT diameter as $\sim 2 \mathrm{~nm}$, we estimate the distance of the electron trap to the outer edge of the $\mathrm{Cr}$ layer as $\sim 0.2 \mathrm{~nm}$, placing the trap just outside the nanotube as expected. Taking the degeneracy ratio $g_{1} / g_{2}$ $\sim 1$ in Eq. (1), one can estimate the offset parameter $V_{0}$ $\sim 2 \mathrm{~V}$. At $V_{g}=0$, this gives an energy difference between the trap state and the nanotube Fermi level as $E_{t}-E_{f} \sim 42 \mathrm{meV}$.

We note that not all FETs in our experiments show clearly resolvable discrete current switching, but most devices exhibit excess fluctuations without resolvable transitions likely due to the many distributed electron traps. This may be one explanation for the previously observed excess noise in open-air nanotubes. ${ }^{7}$ This noise exposes a major challenge to the potential application of carbon nanotubes in electronic devices and it suggests that effort is needed to reliably eliminate traps near the nanotube channel for low noise devices. Electron traps near the interface between the gate dielectrics and the nanotubes contribute most to the random telegraph noise. Optimized gate dielectric processing may enable control of trap distributions in the future.

In summary, we have investigated the electrical current fluctuations of coaxial $p$ - and $n$-type SWCNT FETs. A discrete random telegraph signal due to the perturbation of electron traps near the conducting channel is clearly indicated. The sensitivity of SWCNTs to a single charge changing event at room temperature may provide opportunities for realizing single biomolecule sensors based on suitably fabricated SWCNT FETs. SWCNT FETs with electron traps may also prove useful for single-spin detection. ${ }^{15,16}$

${ }^{1}$ S. J. Tans, A. R. M. Verschueren, and C. Dekker, Nature (London) 393, 49 (1998).

${ }^{2}$ R. Matel, T. Schmidt, H. R. Shea, T. Hertel, and Ph. Avouris, Appl. Phys. Lett. 73, 2447 (1998).

${ }^{3}$ P. G. Collins, K. Bradley, M. Ishigami, and A. Zettl, Science 287, 1801 (2000).

${ }^{4}$ J. Kong, N. R. Franklin, C. Zhou, M. G. Chapline, S. Peng, K. Cho, and H. Dai, Science 287, 622 (2000).

${ }^{5}$ Carbon Nanotubes: Synthesis, Structure, Properties, and Applications, edited by M. S. Dresselhaus, G. Dresselhaus, and P. Avouris (Springer, Berlin, 2001).

${ }^{6}$ P. G. Collins, M. S. Fuhrer, and A. Zettl, Appl. Phys. Lett. 76, 894 (2000). ${ }^{7}$ H. Ouacha, M. Willander, H. Y. Yu, Y. W. Park, M. S. Kabir, S. H. M. Persson, L. B. Kish, and A. Ouacha, Appl. Phys. Lett. 80, 1055 (2002).

${ }^{8}$ L. Roschier, R. Tarkiainen, M. Ahlskog, M. Paalanen, and P. Hakonen, Appl. Phys. Lett. 78, 3295 (2001).

${ }^{9}$ M. S. Fuhrer, B. M. Kim, T. Durkop, and T. Brintlinger, Nano Lett. 2, 755 (2002).

${ }^{10}$ H. B. Peng, T. G. Ristroph, G. M. Schurmann, G. M. King, J. Yoon, V. Narayanamurti, and J. A. Golovchenko, Appl. Phys. Lett. 83, 4238 (2003).

${ }^{11}$ H. B. Peng and J. A. Golovchenko, Appl. Phys. Lett. 84, 5428 (2004).

${ }^{12}$ H. B. Peng and J. A. Golovchenko, e-print cond-mat/0508670.

${ }^{13}$ Haibing Peng, Ph.D. thesis, Harvard University, 2004.

${ }^{14}$ K. S. Ralls, W. J. Skocpol, L. D. Jackel, R. E. Howard, L. A. Fetter, R. W. Epworth, and D. M. Tennant, Phys. Rev. Lett. 52, 228 (1984).

${ }^{15}$ M. Xiao, I. Martin, E. Yablonovitch, and H. W. Jiang, Nature (London) 430, 435 (2004).

${ }^{16}$ The initial submission of this letter was in August 2005. While the letter was in preparation a related study in low temperature appeared in the literature: F. Liu, M. Bao, H. Kim, K. L. Wang, C. Li, X. Liu, and C. Zhou, Appl. Phys. Lett. 86, 163102 (2005). 